\title{
Impact of Continuous Erythropoietin Receptor Activator on Selected Biomarkers of Cardiovascular Disease and Left Ventricle Structure and Function in Chronic Kidney Disease
}

\author{
Piotr Bartnicki, ${ }^{1}$ Jacek Rysz, ${ }^{1}$ Beata Franczyk, ${ }^{1}$ Zbigniew Baj, ${ }^{2}$ and Ewa Majewska ${ }^{2}$ \\ ${ }^{1}$ Department of Nephrology, Hypertension and Family Medicine, Medical University of Lodz, 90-549 Lodz, Poland \\ ${ }^{2}$ Department of Pathophysiology and Clinical Immunology, Medical University of Lodz, 91-647 Lodz, Poland \\ Correspondence should be addressed to Piotr Bartnicki; piotr.bartnicki@umed.lodz.pl
}

Received 25 November 2015; Revised 2 February 2016; Accepted 4 February 2016

Academic Editor: Aramati B. M. Reddy

Copyright (C) 2016 Piotr Bartnicki et al. This is an open access article distributed under the Creative Commons Attribution License, which permits unrestricted use, distribution, and reproduction in any medium, provided the original work is properly cited.

\begin{abstract}
Background. Cardiovascular morbidity and mortality are very high in patients with chronic kidney disease (CKD). The purpose of this study is to evaluate the impact of continuous erythropoietin receptor activator (CERA) on selected biomarkers of cardiovascular disease, left ventricle structure, and function in CKD. Material and Methods. Peripheral blood was collected from 25 CKD patients before and after CERA treatment and 20 healthy subjects. In serum samples, we assessed inflammatory markers (IL-1 $\beta$, TNF-RI, TNF-RII, sFas, sFasL, MMP-9, TIMP-1, and TGF- $\beta 1$ ), endothelial dysfunction markers (sE-selectin, sICAM-1, and sVCAM-1), and volume-related marker (NT-proBNP). All subjects underwent echocardiography and were evaluated for selected biochemical parameters (Hb, creatinine, and CRP). Results. Evaluated biomarkers and echocardiographic parameters of left ventricle structure were significantly increased but left ventricle EF was significantly decreased in CKD patients compared to controls. After CERA treatment, we observed a significant increase of $\mathrm{Hb}$ and left ventricle EF and a significant decrease of NT-proBNP and MMP-9. There was a significant negative correlation between $\mathrm{Hb}$ and TNF-RI, sICAM-1, and IL-1 $\beta$. Conclusions. Our results indicate that selected biomarkers related to cardiovascular risk are significantly increased in CKD patients compared to controls. CERA treatment has anti-inflammatory action, diminishes endothelial dysfunction, and improves left ventricle function in these patients.
\end{abstract}

\section{Introduction}

Cardiovascular diseases (CVD) are significant causes of morbidity and mortality all over the world [1]. Well-known traditional risk factors of CVD are diabetes, hypertension, obesity, and hyperlipidemia. These factors do not fully explain the increase of CVD in patients with chronic kidney disease (CKD) [2] and a very high risk of cardiovascular death, especially in later stages of CKD $[3,4]$. Literature data indicate that pathology, manifestations, complications, and management of CVD differ in CKD patients. Standard therapeutic interventions targeted at traditional risk factors of CVD, that are successful in the general population, are ineffective to lower CVD events and mortality in CKD [5]. It is thought that nontraditional risk factors of CVD play a very important role in pathogenesis of premature atherosclerosis and cardiovascular complications in these patients [2]. The most common nontraditional risk factors of CVD in CKD patients are anemia, calcium/phosphorus disorders, hyperparathyroidism, and malnutrition. It is possible that early therapeutic approaches targeting traditional and nontraditional CVD risk factors may prevent CVD events in CKD, but the information concerning CVD, biomarkers, and treatment in early stages of CKD is limited. Recent studies indicate the role of chronic inflammation, oxidative stress, endothelial dysfunction, and immune cell apoptosis in the pathogenesis of accelerated atherosclerosis and CVD in CKD patients $[6,7]$. Some biomarkers of chronic inflammation such as interleukin 1 beta (IL-1 $\beta$ ), tumor necrosis factoralpha (TNF- $\alpha$ ), oxidative stress (reactive oxygen species, antioxidant enzymes), endothelial dysfunction (asymmetric dimethylarginine, soluble forms of adhesion molecules), and 
apoptosis (Fas, Fas ligand) are well known [8-12]. For better understanding of the pathogenesis of CVD in CKD it is necessary to look for new biomarkers. Recent studies have proposed soluble TNF receptor type II (TNF-RII) and tissue inhibitor of metalloproteinase-1 (TIMP-1) as new biomarkers of chronic inflammation [13] and metalloproteinase-9 (MMP-9), soluble Fas (sFas), soluble Fas ligand (sFasL), soluble intercellular adhesion molecule-1 (sICAM-1), and transforming growth factor-beta 1 (TGF- $\beta 1$ ) as new markers of CVD risk in CKD patients [14-16]. A very important, nontraditional CVD risk factor in CKD patients is anemia [17]. Especially in the later stages of CKD, anemia reduces the quality of life and may increase oxidative stress, endothelial dysfunction, and immune cell apoptosis and consequently may be involved in pathogenesis of premature atherosclerosis and CVD [18]. Anemia treatment with erythropoietin (EPO) in CKD patients seems to have a pleiotropic effect and may reduce chronic inflammation, oxidative stress, and immune cell apoptosis [19]. Our latest studies showed that anemia treatment with methoxy polyethylene glycol-epoetin beta (CERA), a long half-life EPO, in CKD patients may inhibit oxidative stress and immune cell apoptosis [20, 21]. Finally in consequence of these pleiotropic EPO effects it is thought that timely correction of anemia may lead to a significant improvement in the cardiovascular outcome in CKD patients [22]. The purpose of this study was to evaluate the impact of CERA treatment by using methoxy polyethylene glycolepoetin (MPG-EPO) beta on selected CVD risk biomarkers, especially of inflammation and endothelial dysfunction, and left ventricle structure and function, in nondialyzed CKD patients.

\section{Materials and Methods}

2.1. Patient Population. Thirty-five patients (20 men and 15 women, median age 59 years, range 45-69) with CKD were enrolled in the study. All patients were in stage IV of CKD with an estimated glomerular filtration rate (eGFR) of 15$29 \mathrm{~mL} / \mathrm{min}$, calculated according to the Modification of Diet in Renal Disease (MDRD) equation. They were on conservative treatment and had never undergone dialysis. Patients with age over 70 years, diabetes, blood transfusion in the past 3 months, acute infection, chronic infection (hepatitides $\mathrm{B}$ and $\mathrm{C}$ ), autoimmune disease, immunosuppressive therapy, increased C-reactive protein (CRP) over $25 \mathrm{mg} / \mathrm{L}$, or a history of malignancy were excluded from the study. The relatively small number of patients, due to many exclusion criteria, was sufficient to give statistical power (70-100\%). Causes of CKD were primary hypertension with chronic kidney disease (40\%), chronic tubule-interstitial nephritis (23\%), chronic glomerulonephritis (20\%), and polycystic kidney disease (17\%).

The control group included 20 healthy volunteers (12 men and 8 women, median age 56, range 48-63) without CKD (normal eGFR, hemoglobin concentration, urine analysis, and kidney ultrasound). All investigated subjects had not been smoking for at least 5 years.

All CKD patients had anemia with hemoglobin $(\mathrm{Hb})$ concentration lower than $10 \mathrm{~g} / \mathrm{dL}$. After exclusion of bleeding, iron deficiency, hemolysis, infection, and severe secondary hyperparathyroidism, they received a subcutaneous injection of MPG-EPO (Mircera, Roche, Basel, Switzerland) in a dose of $0.6 \mu \mathrm{g} / \mathrm{kg}$ once monthly. Treatment was continued until reaching the target $\mathrm{Hb}$ concentration of $11-12 \mathrm{~g} / \mathrm{dL}$, achieved by 25 patients, who were enrolled in the next part of the study. Ten patients started dialysis treatment before they achieved target $\mathrm{Hb}$ and were excluded from the study. Average treatment time was 227 days (from 108 to 428 days), and the average MPG-EPO dose was $50 \mu \mathrm{g} / \mathrm{month}$ (from 30 to $75 \mu \mathrm{g})$.

The study was approved by the Ethics Committee of Research of the Medical University of Lodz, number RNN/97/09/KB. Only patients who signed informed consent were included in the study.

\subsection{Measurement of Biochemical Parameters and Biomarkers.} Peripheral blood was drawn from CKD patients twice (before treatment with MPG-EPO and after achieving target $\mathrm{Hb}$ concentration) and from healthy volunteers once. Overnight fasting venous blood was collected into sodium citrate tubes and centrifuged at $4^{\circ} \mathrm{C}$ with a speed of $1000 \mathrm{~g}$ for 20 minutes. The obtained serum samples for biomarker assessment were frozen and stored at $-80^{\circ} \mathrm{C}$ until processed. We measured serum concentration of IL- $1 \beta$, soluble TNF receptors I and II (TNF-RI, TNF-RII), sFas, sFasL, soluble endothelial leukocyte adhesion molecule-1 (sE-selectin), sICAM-1, soluble human vascular cell adhesion molecule-1 (sVCAM-1), MMP9, TIMP-1, and TGF- $\beta 1$ using commercially available human Quantikine ELISA Kits (R\&D Systems, Minneapolis, USA). Sample collection and storage, reagent preparation, assay procedure, and calculation of results were performed according to the manufacturer's instructions. The mean minimum detectable doses of assessed biomarkers, according to the manufacturer, were as follows: IL- $\beta 1$, less than $1 \mathrm{pg} / \mathrm{mL}$; sTNF-RI, $0.77 \mathrm{pg} / \mathrm{mL}$; sTNF-RII, $0.6 \mathrm{pg} / \mathrm{mL}$; sFas, $20 \mathrm{pg} / \mathrm{mL}$; sFasL, $2.6 \mathrm{pg} / \mathrm{mL}$; sE-selectin, $0.009 \mathrm{ng} / \mathrm{mL}$; sVCAM-1, 0.6 ng/mL; sICAM-1, 0.096 ng/mL; MMP-9, 0.156 ng/mL; TIMP1 , less than $0.08 \mathrm{ng} / \mathrm{mL}$; TGF- $\beta 1,4.61 \mathrm{pg} / \mathrm{mL}$. The biochemical parameters, serum $\mathrm{Hb}$, creatinine, $\mathrm{C}$-reactive protein (CRP), $\mathrm{N}$-terminal pro-brain natriuretic peptide (NT-proBNP) concentration, were assessed using standard techniques in a local laboratory.

2.3. Evaluation of CVD. All CKD patients were diagnosed with hypertension and received the following hypotensive drugs: ACE inhibitors, AT-II receptor blockers, loop diuretics, beta blockers, and calcium channel blockers. The majority of them were diagnosed with chronic coronary heart disease and underwent percutaneous coronoplasty with stent implantation. Some of them presented clinical symptoms of chronic heart failure, such as shortness of breath, tiredness, and peripheral edema. To evaluate left ventricle structure and function, echocardiography was performed twice in the CKD patients (before MPG-EPO treatment and after achieving target $\mathrm{Hb}$ concentration) and once in the control group. Echocardiographic examination was performed in accordance with the recommendations of the ESC Section of Echocardiography of 2009 using an Aloka ProSound Alpha 
TABLE 1: Results of biochemical parameters in CKD patients and control group.

\begin{tabular}{lccc}
\hline Biochemical parameters & $\begin{array}{c}\text { CKD patients before treatment } \\
(n=25)(\mathrm{Me} ; 25-75 \%)\end{array}$ & $\begin{array}{c}\text { CKD patients after treatment } \\
(n=25)(\mathrm{Me} ; 25-75 \%)\end{array}$ & $\begin{array}{c}\text { Control group } \\
(n=20)(\mathrm{Me} ; 25-75 \%)\end{array}$ \\
\hline $\mathrm{Hb}(\mathrm{g} / \mathrm{dL})$ & $9.1(8.7-10.0)^{*}$ & $11.6(11.1-12.1)^{* *}$ & $14.4(13.3-14.9)$ \\
Creatinine $(\mu \mathrm{mol} / \mathrm{L})$ & $269(233-340)^{*}$ & $271(223-348)^{*}$ & $90(77-108)$ \\
eGFR $(\mathrm{MDRD})\left(\mathrm{mL} / \mathrm{min} / 1.73 \mathrm{~m}^{2}\right)$ & $19.6(15.5-22.8)^{*}$ & $18(15-24.2)^{*}$ & $65(63-74)$ \\
$\mathrm{CRP}(\mathrm{mg} / \mathrm{L})$ & $18(14.1-22.8)^{*}$ & $16(12.2-20.4)^{*}$ & $4.5(3.7-6.9)$ \\
NT-proBNP $(\mathrm{pg} / \mathrm{mL})$ & $355.6(282.3-432.7)^{*}$ & $302(182.1-401.3)^{* \circ}$ & $75(49-101)$ \\
\hline
\end{tabular}

${ }^{*} p<0.05$ versus control group; ${ }^{\circ} p<0.05$ versus $\mathrm{CKD}$ patients before treatment.

$\mathrm{CKD}=$ chronic kidney disease, $(\mathrm{Me} ; 25-75 \%)=$ median and interquartile range, $\mathrm{Hb}=$ hemoglobin, eGFR $(\mathrm{MDRD})=$ estimated glomerular filtration rate (Modification of Diet in Renal Disease equation), CRP $=$ C-reactive protein, and NT-proBNP $=$ N-terminal pro-brain natriuretic peptide.

TABLE 2: Results of assessed biomarkers in CKD patients and control group.

\begin{tabular}{lccc}
\hline Biomarkers & $\begin{array}{c}\text { CKD patients before treatment } \\
(n=25)(\mathrm{Me} ; 25-75 \%)\end{array}$ & $\begin{array}{c}\text { CKD patients after treatment } \\
(n=25)(\mathrm{Me} ; 25-75 \%)\end{array}$ & $\begin{array}{c}\text { Control group } \\
(n=20)(\mathrm{Me} ; 25-75 \%)\end{array}$ \\
\hline IL-1 $\beta(\mathrm{pg} / \mathrm{mL})$ & $2.78(2.00-3.36)^{*}$ & $2.69(2.48-3.15)^{*}$ & $1.25(0.89-1.65)$ \\
TNF-RI $(\mathrm{pg} / \mathrm{mL})$ & $4644(4156-5677)^{*}$ & $4979(4236-6667)^{*}$ & $1151(930-1332)$ \\
TNF-RII $(\mathrm{pg} / \mathrm{mL})$ & $8010(7298-8689)^{*}$ & $9180(8845-9908)^{* \circ}$ & $2351(1900-2600)$ \\
sFasL $(\mathrm{pg} / \mathrm{mL})$ & $85.9(68.0-109.9)^{*}$ & $94.3(75.6-103.8)^{*}$ & $47.0(30.5-80.0)$ \\
sFas $(\mathrm{pg} / \mathrm{mL})$ & $3272(2734-3799)^{*}$ & $3206(2826-3715)^{*}$ & $465.0(368.0-645.0)$ \\
sE-selectin $(\mathrm{ng} / \mathrm{mL})$ & $28.3(24.2-33.6)^{*}$ & $33.5(26.0-36.2)^{*}$ & $15.5(11.7-22.9)$ \\
sICAM-1 $(\mathrm{ng} / \mathrm{mL})$ & $402.0(336.0-504.0)^{*}$ & $466.0(355.0-518.0)^{*}$ & $212.0(178.0-228.0)^{*}$ \\
TIMP-1 $(\mathrm{ng} / \mathrm{mL})$ & $203.0(186.5-258.5)^{*}$ & $2600(1720-4129)^{* \circ}$ & $110.0(91.0-131.0)$ \\
sVCAM-1 $(\mathrm{ng} / \mathrm{mL})$ & $2380(1326-2790)^{*}$ & $586.0(450.0-764.0)^{* \circ}$ & $840.0(760.0-1252.0)$ \\
MMP-9 $(\mathrm{ng} / \mathrm{mL})$ & $1062(665-1455)^{*}$ & $21.2(16.5-29.3)^{*}$ & $280.0(217.0-292.0)$ \\
TGF- $\beta 1(\mathrm{pg} / \mathrm{mL})$ & $21.4(19.0-30.1)^{*}$ & & $11.6(9.7-15.5)$ \\
\hline
\end{tabular}

${ }^{*} p<0.05$ versus control group; ${ }^{\circ} p<0.05$ versus CKD patients before treatment.

$\mathrm{CKD}=$ chronic kidney disease, $(\mathrm{Me} ; 25-75 \%)=$ median and interquartile range, IL-1 $\beta=$ interleukin $1 \beta$, TNF-RI = soluble tumor necrosis factor receptor I, TNF-RII = soluble tumor necrosis factor receptor II, sFasL = soluble Fas ligand, sFas = soluble Fas, sICAM-1 = soluble intercellular adhesion molecule-1, TIMP-1 = tissue inhibitor of metalloproteinase-1, sVCAM-1 = soluble human vascular cell adhesion molecule-1, MMP-9 = metalloproteinase-9, and TGF- $\beta 1=$ transforming growth factor-beta 1.

camera 10 (Hitachi Aloka Medical, Ltd., Tokyo, Japan) by an experienced cardiologist. Measurements were made in the M-dimensional and two-dimensional 2D presentation. Flow parameters were measured by Doppler: continuous wave (CW), pulse method (PM), and tagged color methods and tissue Doppler imaging. The following echocardiographic parameters were assessed: intraventricular septal diameter (IVSd), left ventricular end systolic diameter (LVESd), left ventricular end diastolic diameter (LVEDd), and left atrial diameter (LA). These measurements were used to evaluate left ventricular ejection fraction (EF), left ventricular mass (LVM), left ventricular hypertrophy (LVH), and left ventricular diastolic dysfunction (LVDD). Diastolic function was assessed by determining the velocities of early (E) and late (A) diastolic transmitral flow, the ratio E-to-A (E/A), deceleration time (DT), isovolumic relaxation time (IVRT), and pulmonary vein flow velocities.

2.4. Statistical Analysis. Data are presented as the median $(\mathrm{Me})$ and interquartile range (Me; $25-75 \%)$. Evaluation of statistical significance was performed with the Wilcoxon signed ranks test for paired data and the Mann-Whitney $U$ test for unpaired data. Correlation between $\mathrm{Hb}$ concentration, evaluated biomarkers, and echocardiographic parameters was performed by calculation of Spearman $r$ correlation coefficient values. Statistical significance was assumed at a $p$ value $<0.05$. The statistical analysis was carried out using the statistical software Statistica (StatSoft, Inc., Tulsa, OK, USA).

\section{Results}

The results concerning biochemical parameters in CKD patients and the control group are shown in Table $1 . \mathrm{Hb}$ concentration and eGFR were significantly lower in CKD patients in comparison to the control group, but serum creatinine, CRP, and NT-proBNP concentrations were significantly higher in CKD patients in comparison to the control group. After MPG-EPO treatment, CKD patients had a significantly higher $\mathrm{Hb}$ concentration and significantly lower serum NTproBNP concentration in comparison to before treatment. MPG-EPO treatment did not change kidney function or serum CRP concentration.

The results of evaluated biomarkers in CKD patients before and after MPG-EPO treatment and the control group are shown in Table 2 and Figure 1. Serum concentration of all assessed biomarkers was significantly higher in CKD patients in comparison to the control group. After MPG-EPO treatment, CKD patients had significantly higher serum TNF-RII 

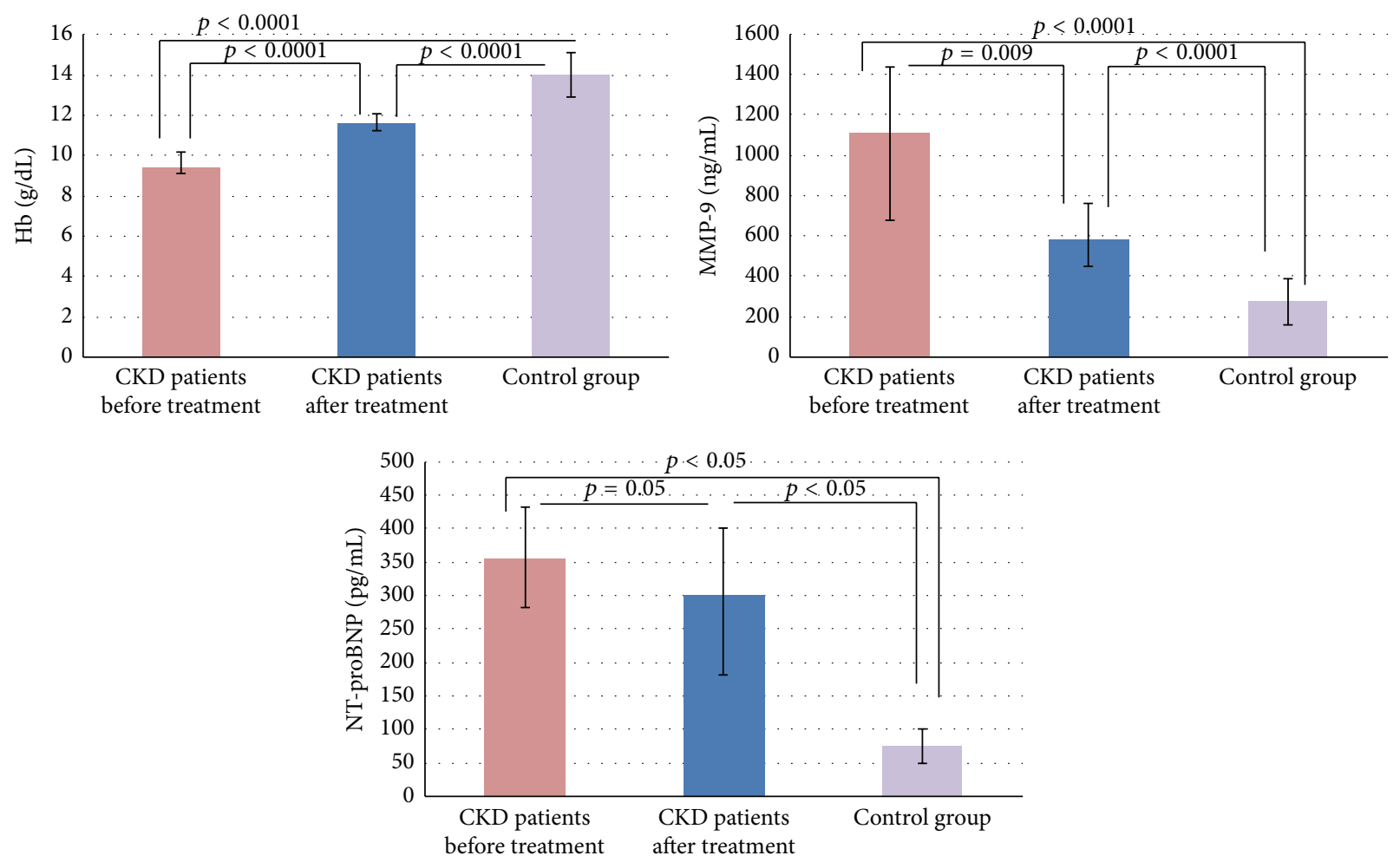

FIGURE 1: Results of serum Hb, MMP-9, and NT-proBNP concentrations in CKD patients and control group. Hb = hemoglobin, MMP-9 = metalloproteinase-9, NT-proBNP = N-terminal pro-brain natriuretic peptide, and CKD = chronic kidney disease.

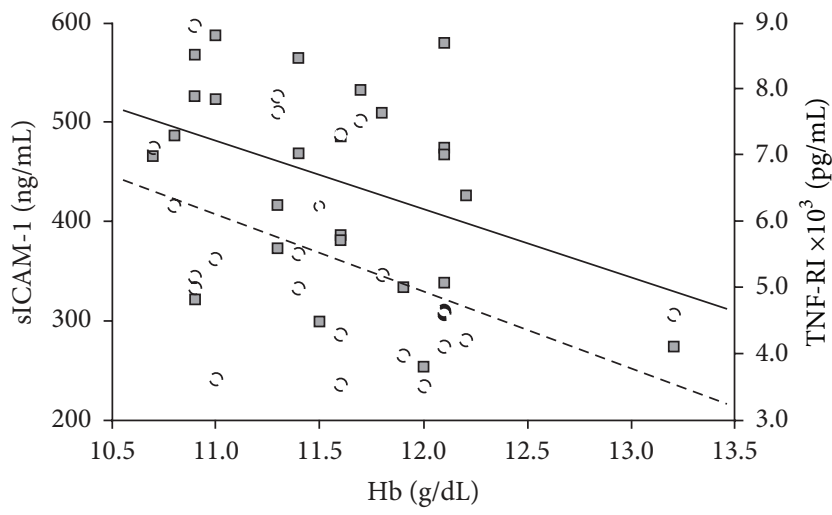

FIGURE 2: Correlation of serum $\mathrm{Hb}$ with TNF-RI and sICAM-1 concentrations in CKD patients. sICAM-1: squares and continuous line; TNF-RI: circles and broken line. $\mathrm{Hb}=$ hemoglobin, TNF$\mathrm{RI}=$ soluble tumor necrosis factor receptor I, sICAM-1 = soluble intercellular adhesion molecule-1, and $\mathrm{CKD}=$ chronic kidney disease.

and sVCAM-1 concentrations, but MMP-9 concentration was significantly lower than before treatment.

Table 3 and Figure 2 show the correlation between $\mathrm{Hb}$ concentration and evaluated biomarkers in CKD patients before and after MPG-EPO treatment. There was a significant negative correlation between $\mathrm{Hb}$ concentration with serum TNF-RI and sICAM-1 concentrations.

The correlations between increase of $\mathrm{Hb}$ concentration expressed as absolute values or as percentages and changes

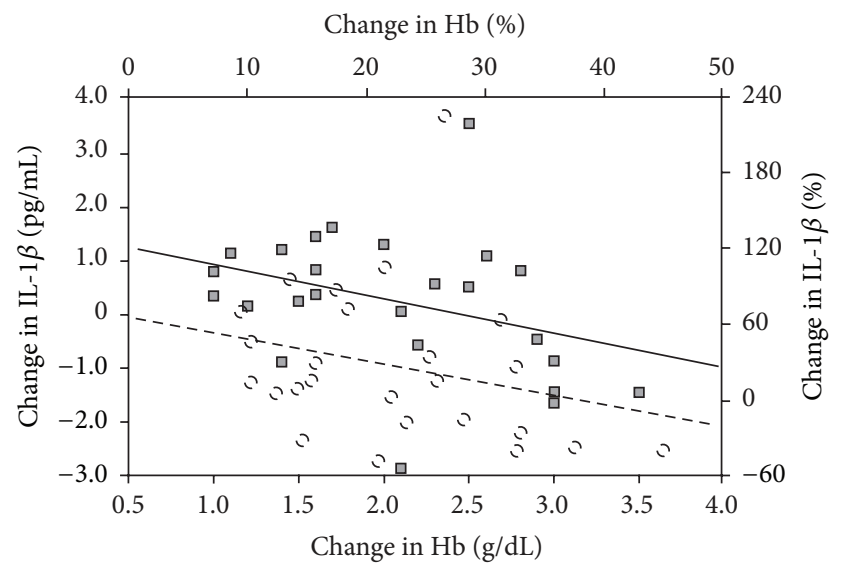

FIgURE 3: Correlation between increase of $\mathrm{Hb}$ concentration expressed as absolute value $(\mathrm{g} / \mathrm{dL})$ or as percentage (\%) and change of plasma IL-1 $\beta$ concentration expressed as absolute value $(\mathrm{pg} / \mathrm{mL})$ or as percentage (\%) in CKD patients. IL-1 beta $(\mathrm{pg} / \mathrm{mL})$ and $\mathrm{Hb}$ $(\mathrm{g} / \mathrm{dL})$ : squares and continuous line; IL-1 beta (\%) and $\mathrm{Hb}(\%)$ : circles and broken line. $\mathrm{Hb}=$ hemoglobin, IL-1 $\beta=$ interleukin 1 beta, and $\mathrm{CKD}=$ chronic kidney disease.

of evaluated biomarkers expressed as absolute values or as percentages in CKD patients are shown in Table 4 and Figure 3. There was a significant negative correlation between increase of $\mathrm{Hb}$ concentration and serum IL- $1 \beta$ concentration.

Evaluated echocardiographic parameters in the study groups are shown in Table 5. All CKD patients had left 
TABLE 3: Correlations between serum $\mathrm{Hb}$ concentration and evaluated biomarkers in CKD patients.

\begin{tabular}{lcc}
\hline Biomarkers & $\begin{array}{c}\text { CKD patients } \\
\text { before treatment } \\
n=25\end{array}$ & $\begin{array}{c}\text { CKD patients } \\
\text { after treatment }\end{array}$ \\
& $\mathrm{Hb}$ & $n=25$ \\
$\mathrm{IL}-1 \beta(\mathrm{pg} / \mathrm{mL})$ & -0.140 & 0.117 \\
sTNF-RI $(\mathrm{pg} / \mathrm{mL})$ & -0.040 & $-0.444^{*}$ \\
sTNF-RII $(\mathrm{pg} / \mathrm{mL})$ & -0.157 & 0.270 \\
sFasL $(\mathrm{pg} / \mathrm{mL})$ & -0.307 & -0.015 \\
sFas $(\mathrm{pg} / \mathrm{mL})$ & -0.196 & -0.164 \\
sE-selectin $(\mathrm{ng} / \mathrm{mL})$ & -0.025 & -0.023 \\
sICAM-1 $(\mathrm{ng} / \mathrm{mL})$ & 0.148 & $-0.403^{*}$ \\
TIMP-1 $(\mathrm{ng} / \mathrm{mL})$ & -0.156 & -0.222 \\
sVCAM-1 $(\mathrm{ng} / \mathrm{mL})$ & 0.116 & 0.083 \\
MMP-9 $(\mathrm{ng} / \mathrm{mL})$ & 0.102 & -0.140 \\
TGF- $\beta 1(\mathrm{pg} / \mathrm{mL})$ & -0.026 & -0.138 \\
\hline
\end{tabular}

Spearman $r$ correlation coefficient values, ${ }^{*} p<0.05$.

$\mathrm{Hb}=$ hemoglobin, $\mathrm{CKD}=$ chronic kidney disease, $\mathrm{IL}-1 \beta=$ interleukin $1 \beta$, TNF-RI = soluble tumor necrosis factor receptor I, TNF-RII = soluble tumor necrosis factor receptor II, sFasL = soluble Fas ligand, $s$ Fas $=$ soluble Fas, sICAM-1 = soluble intercellular adhesion molecule-1, TIMP-1 = tissue inhibitor of metalloproteinase-1, sVCAM-1 = soluble human vascular cell adhesion molecule-1, MMP-9 = metalloproteinase-9, and TGF- $\beta 1=$ transforming growth factor- beta 1 .

ventricular hypertrophy (LVH), and the majority of them had left ventricular diastolic dysfunction (LVDD). Left ventricular ejection fraction (EF) was significantly lower in CKD patients than the control group. After MPG-EPO treatment, $\mathrm{EF}$ in CKD patients was significantly increased in comparison to before treatment but was still significantly lower than in the control group. Other evaluated echocardiographic parameters of left ventricle structure were significantly higher in CKD patients than in the control group, and MPG-EPO treatment did not change them significantly.

Table 6 and Figure 4 show the correlation between $\mathrm{Hb}$ concentration and evaluated echocardiographic parameters of left ventricle structure and function. There was a significant positive correlation between $\mathrm{Hb}$ and $\mathrm{EF}$ and a significant negative correlation between $\mathrm{Hb}$ and LVESd.

\section{Discussion}

IL-1 (IL-1 $\alpha$ and IL-1 $\beta$ ) plays a central role in acute and chronic inflammation [23]. In our study, plasma concentration of the proinflammatory cytokine IL- $1 \beta$ was significantly higher in CKD patients than in the control group. Our result is comparable to data available in the literature where the plasma IL-1 $\beta$ concentration was found elevated in nondialyzed CKD patients as well as in CKD patients on dialysis treatment $[24,25]$. It is well documented that CKD is associated with chronic inflammation and uremic toxins can induce production of IL-1 mainly by monocytes and macrophages [26]. In CKD patients, other proinflammatory cytokines were found to be elevated, such as IL- 6 and TNF- $\alpha$, which

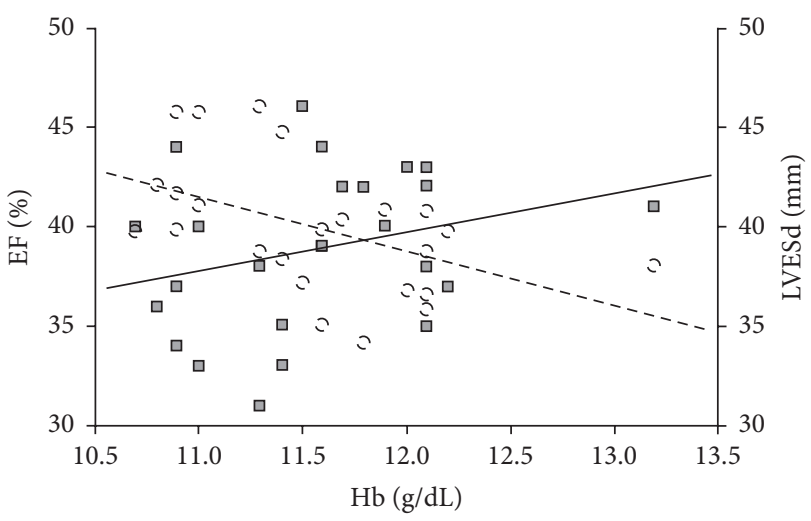

FIgURE 4: Correlation of serum $\mathrm{Hb}$ with EF and LVESd in CKD patients. EF (\%): squares and continuous line; LVESd: circles and broken line. $\mathrm{Hb}=$ hemoglobin, $\mathrm{EF}=$ left ventricular ejection fraction, LVESd = left ventricular end systolic diameter, and CKD $=$ chronic kidney disease.

modify inflammatory and immune reactions [27]. The next step in the inflammatory reaction is increasing expression of cell adhesion molecules (E-selectin, ICAM-1, VCAM-1), responsible for extravasation of leukocytes and activation of metalloproteinases (MMPs). We found in our study significantly elevated plasma sE-selectin, sICAM-1, sVCAM-1, and MMP-9 concentrations in CKD patients in comparison to the control group. Data available in the literature are comparable; Bonomini et al. found elevated serum levels of soluble ICAM1, VCAM-1, and E-selectin in both nondialyzed CKD patients and those under dialysis treatment [28]. Other authors reported elevated levels of these cell adhesion molecules in patients undergoing chronic hemodialysis [11]. High serum concentration of soluble adhesion molecules in CKD patients may indicate vascular endothelial cell activation, which is involved in the pathogenesis of atherosclerosis and CVD in these patients [29]. Elevated plasma levels of sICAM-1 were strongly associated with CVD and could be considered as a novel biomarker of CVD [30]. Data in the literature regarding serum MMP-9 concentration, where the authors found significantly lower MMP-9 concentration in CKD patients in comparison to the control group [31], are opposite to our results. IL-1 and other proinflammatory cytokines induce synthesis of TIMP-1, an inhibitor of MMP-9, which we found significantly elevated in CKD patients in comparison to the control group. Similar data have been reported by other authors $[14,31]$. The latest data in the literature indicate elevated serum MMP-9 concentration as a new biomarker of CVD in CKD [2] and TIMP-1 concentration as a new marker of inflammation in CKD patients [13]. In our study, plasma TGF- $\beta 1$ concentration was significantly higher than in the control group. TGF- $\beta 1$ is well known as a promoter of extracellular matrix synthesis. In experimental chronic renal failure, a correlation was found between elevated levels of TGF- $\beta 1$ and TNF- $\alpha$ with cardiac fibrosis [15], which may indicate the involvement of these biomarkers in pathogenesis of CVD in CKD. The next proinflammatory cytokine is TNF, serum concentration of which is elevated in CKD. Literature 
TABLE 4: Correlation between increase of $\mathrm{Hb}$ concentration expressed as absolute values $(\Delta)$ or as percentages (\%) and change of evaluated biomarkers expressed as absolute values $(\Delta)$ or as percentages $(\%)$ in CKD patients.

\begin{tabular}{lccc}
\hline $\begin{array}{l}\text { Biomarkers }(\Delta) \text { after-before } \\
\text { treatment }\end{array}$ & $\begin{array}{c}\mathrm{Hb}(\Delta) \text { after-before } \\
\text { treatment }\end{array}$ & $\begin{array}{c}\text { Biomarkers }(\%) \\
{[(\text { after-before }) / \text { before treatment }] \times 100}\end{array}$ & $\begin{array}{c}\text { Hb }(\%) \\
{[(\text { after-before }) / \text { before treatment }] \times 100}\end{array}$ \\
\hline IL-1 $\beta$ & $-0.511^{*}$ & IL-1 $\beta$ & $-0.484^{*}$ \\
TNF-RI & -0.061 & TNF-RI & -0.081 \\
TNF-RII & -0.250 & TNF-RII & -0.276 \\
sFasL & -0.070 & sFasL & -0.088 \\
sFas & -0.096 & sFas & -0.093 \\
sE-selectin & -0.128 & sE-selectin & -0.105 \\
sICAM-1 & 0.144 & sICAM-1 & 0.125 \\
TIMP-1 & 0.040 & TIMP-1 & 0.066 \\
sVCAM-1 & -0.074 & sVCAM-1 & 0.013 \\
MMP-9 & 0.094 & MMP-9 & 0.055 \\
TGF- $\beta 1$ & -0.124 & TGF- $\beta 1$ & -0.098 \\
\hline
\end{tabular}

Spearman $r$ correlation coefficient values, ${ }^{*} p<0.05$.

$\mathrm{Hb}=$ hemoglobin, $\mathrm{CKD}=$ chronic kidney disease, IL-1 $\beta=$ interleukin $1 \beta$, TNF-RI $=$ soluble tumor necrosis factor receptor I, TNF-RII $=$ soluble tumor necrosis factor receptor II, $\mathrm{sFasL}=$ soluble Fas ligand, $\mathrm{sFas}=$ soluble Fas, sICAM-1 = soluble intercellular adhesion molecule-1, TIMP- $1=$ tissue inhibitor of metalloproteinase-1, sVCAM-1 = soluble human vascular cell adhesion molecule-1, MMP-9 = metalloproteinase- 9 , and TGF- $\beta 1$ = transforming growth factorbeta 1.

TABLE 5: Echocardiographic parameters in CKD patients and control group.

\begin{tabular}{lccc}
\hline & $\begin{array}{c}\text { CKD patients before treatment } \\
(n=25)(\mathrm{Me} ; 25-75 \%)\end{array}$ & $\begin{array}{c}\text { CKD patients after treatment } \\
(n=25)(\mathrm{Me} ; 25-75 \%)\end{array}$ & $\begin{array}{c}\text { Control group } \\
(n=20)(\mathrm{Me} ; 25-75 \%)\end{array}$ \\
\hline IVSd $(\mathrm{mm})$ & $16(15.7-18.3)^{*}$ & $15(11.5-18.1)^{*}$ & $12(10.6-13.4)$ \\
LVM $(\mathrm{g})$ & $287.8(217.7-357.9)^{*}$ & $279.2(183.2-355.2)^{*}$ & $206(163.8-248.2)$ \\
LVESd $(\mathrm{mm})$ & $41(34.8-47.2)^{*}$ & $39.9(32.7-45.1)^{*}$ & $36(30.1-41.9)$ \\
LVEDd $(\mathrm{mm})$ & $48.5(41.8-55.2)^{*}$ & $47.1(41.5-52.7)^{*}$ & $40(32.6-47.4)$ \\
LA $(\mathrm{mm})$ & $41.9(39.2-44.6)^{*}$ & $41.3(37.1-43.5)^{*}$ & $31(28.8-33.2)$ \\
EF $(\%)$ & $35(30-42)^{*}$ & $42(35-48)^{*}$ & $60(51-69)$ \\
LVH $(\%)$ & $100^{*}$ & $100^{*}$ & 20 \\
LVDD $(\%)$ & $96.7^{*}$ & $91.6^{*}$ & 27 \\
\hline
\end{tabular}

${ }^{*} p<0.05$ versus control group; ${ }^{\circ} p<0.05$ versus CKD patients before treatment.

$\mathrm{CKD}=$ chronic kidney disease, IVSd = intraventricular septal diameter, LVM = left ventricular mass, LVESd = left ventricular end systolic diameter, LVEDd $=$ left ventricular end diastolic diameter, $\mathrm{LA}=$ left atrial diameter, $\mathrm{EF}=$ left ventricular ejection fraction, $\mathrm{LVH}=$ left ventricular hypertrophy, and LVDD = left ventricular diastolic dysfunction.

data indicate that TNF elevation correlates with high concentration of soluble TNF receptors (TNF-RI and TNF-RII), which are thought to be novel biomarkers of inflammation in CKD, especially TNF-RII [2]. We found significantly higher serum TNF-RI and TNF-RII concentrations in CKD patients in comparison to the control group. Similar data were obtained by other authors in nondialyzed CKD patients and in patients under chronic hemodialysis treatment [32]. The Fas/FasL system is related to the endothelial cell apoptosis and inflammatory responses in atherosclerotic plaques [33]. Soluble forms of this system ( $\mathrm{sFas}$ and $\mathrm{sFasL}$ ) are proposed as novel biomarkers of vascular damage and high cardiovascular risk. Blanco-Colio et al. showed that increased sFas and decreased sFasL were connected with high cardiovascular risk [16]. In our study, plasma sFas and sFasL concentrations were significantly higher in CKD patients in comparison to the control group. The results of our study indicate that CKD is strongly associated with inflammation, immune system dysregulation, endothelial dysfunction, and vascular damage. All these are involved in pathogenesis of accelerated atherosclerosis and could be responsible for the high rate of CVD in patients with CKD. Patients with CKD enrolled in our study had CVD such as hypertension, chronic coronary heart disease, and chronic heart failure. They had elevated serum NT-proBNP concentration and disorders of left ventricle structure and function in comparison to the control group. Erythropoiesis-stimulating agents (ESAs) are widely used in CKD patients to correct anemia. It is documented in the literature that ESAs beside their main action could have a pleiotropic effect on various cells [34]. It was shown that ESAs could have anti-inflammatory, antioxidant, and antiapoptotic action [19-21]. Anemia correction during ESA treatment can improve heart function [35]. In our study, CKD patients after MPG-EPO treatment achieved a target $\mathrm{Hb}$ concentration which was significantly higher in comparison to before treatment. We observed improvement of left ventricle function 
TABLE 6: Correlation between serum Hb concentration and echocardiographic parameters of left ventricle structure and function in CKD patients.

\begin{tabular}{lcc}
\hline $\begin{array}{l}\text { Echocardiographic } \\
\text { parameters }\end{array}$ & $\begin{array}{c}\text { CKD patients } \\
\text { before treatment } \\
(n=25)\end{array}$ & $\begin{array}{c}\text { CKD patients } \\
\text { after treatment } \\
(n=25)\end{array}$ \\
\hline IVSd (mm) & -0.742 & $\mathrm{Hb}$ \\
LVM (g) & -0.953 & -0.425 \\
LVESd (mm) & -0.768 & -0.613 \\
LVEDd (mm) & -0.748 & $-0.543^{*}$ \\
LA (mm) & -0.774 & -0.350 \\
EF $(\%)$ & 0.949 & -0.498 \\
\hline
\end{tabular}

Spearman $r$ correlation coefficient values, ${ }^{*} p<0.05$.

$\mathrm{Hb}=$ hemoglobin, $\mathrm{CKD}=$ chronic kidney disease, IVSd = intraventricular septal diameter, $L V M=$ left ventricular mass, LVESd = left ventricular end systolic diameter, LVEDd = left ventricular end diastolic diameter, $\mathrm{LA}=$ left atrial diameter, and $\mathrm{EF}=$ left ventricular ejection fraction.

in CKD patients after MPG-EPO treatment as reflected in a significant increase of $\mathrm{EF}$ and a significant decrease of serum NT-proBNP concentration. Echocardiographic parameters of left ventricle structure did not change significantly after treatment, which may be connected with the relatively short average treatment time. Further analysis of the correlation between $\mathrm{Hb}$ and echocardiographic parameters of left ventricle structure and function showed a significant positive correlation between $\mathrm{Hb}$ and $\mathrm{EF}$ and a significant negative correlation between $\mathrm{Hb}$ and LVESd. These results indicate that CERA treatment may improve left ventricle function in CKD patients. After MPG-EPO treatment, we observed significantly increased serum TNF-RII and sVCAM-1 concentrations and a significantly decreased serum MMP-9 concentration. The results concerning TNF-RII and sVCAM1 are unclear. On the one hand, they may indicate that anemia treatment with MPG-EPO enhances inflammation and endothelial dysfunction. We found only one paper in the available literature concerning conversion from short half-life ESAs to MPG-EPO, where the authors noted a significantly increased plasma VCAM concentration after conversion to MPG-EPO (Mircera) [36]. On the other hand, further analysis of our results showed a significant negative correlation between $\mathrm{Hb}$ concentration and serum TNF-RI as well as sICAM-1 concentration after MPG-EPO treatment. A significant negative correlation between increase of $\mathrm{Hb}$ concentration and change of serum IL- $1 \beta$ concentration was also found. Our results concerning serum concentrations of MMP-9, TNF-RI, sICAM-1, and IL- $1 \beta$ indicate that CERA treatment using MPG-EPO beside the main action to correct anemia can improve left ventricle function, demonstrate antiinflammatory action, and may improve endothelial function in nondialyzed CKD patients. In the available literature, most data about ESAs concern the short half-life ESAs used mainly 1-3 times weekly in CKD patients. It is well documented that short half-life ESAs can prevent accelerated atherosclerosis and cardiovascular complications in CKD $[19,35]$. However, high doses of the short half-life ESAs were associated with elevated inflammatory biomarkers [37] and higher mortality risk [38]. In our study, we used the long half-life ESA MPGEPO (CERA), which can be injected once or twice monthly only [39]. We achieved long and slow action of the used ESA with slow correction of anemia, without impairment of renal function and at the same time a positive impact on the evaluated biomarkers of cardiovascular risk and left ventricle function. Anemia treatment with CERA in nondialyzed CKD patients may have significant potential to prevent or reduce cardiovascular complications in these patients. In an animal model, it was shown that chronic treatment with CERA protects against cardiac fibrosis [40]. However, a large-scale longitudinal clinical study should be conducted to determine whether the improvement of inflammation and endothelial dysfunction biomarkers caused by CERA treatment has an impact on better cardiovascular outcome in CKD patients. Further research may also determine whether CERA treatment already in early stages of CKD could improve the CVD outcome in these patients.

\section{Conclusions}

The results of our study indicate that all evaluated biomarkers of inflammation and endothelial dysfunction are significantly increased in nondialyzed CKD patients compared to controls. From our study, the most important new inflammatory biomarkers of CVD risk in CKD patients seem to be TNFRI, sICAM-1, and MMP-9. CERA treatment by use of MPGEPO has anti-inflammatory action, may diminish endothelial dysfunction, and improves left ventricle function in CKD patients. These beneficial effects of MPG-EPO treatment in these patients could be due to the correction of anemia and improvement of blood circulation and oxygenation of tissue and organs. CERA treatment could be considered as a novel therapeutic approach to prevent some mechanisms involved in pathogenesis of the atherosclerotic process and CVD in CKD patients. Further intensive research on large groups of patients is needed to confirm the positive correlation between CERA treatment and the decrease of cardiovascular complications in CKD patients.

\section{Conflict of Interests}

The authors declare that there is no conflict of interests regarding the publication of this paper.

\section{Acknowledgment}

This work was supported by Medical University of Lodz.

\section{References}

[1] G. A. Roth, M. D. Huffman, A. E. Moran et al., "Global and regional patterns in cardiovascular mortality from 1990 to 2013," Circulation, vol. 132, no. 17, pp. 1667-1678, 2015.

[2] Q. Y. Yao, R. Pecoits-Filho, B. Lindholm, and P. Stenvinkel, "Traditional and non-traditional risk factors as contributors to atherosclerotic cardiovascular disease in end-stage renal 
disease," Scandinavian Journal of Urology and Nephrology, vol. 38, no. 5, pp. 405-416, 2004.

[3] M. J. Sarnak, A. S. Levey, A. C. Schoolwerth et al., "Kidney disease as a risk factor for development of cardiovascular disease: a statement from the American Heart Association Councils on Kidney in Cardiovascular Disease, High Blood Pressure Research, Clinical Cardiology, and Epidemiology and Prevention," Hypertension, vol. 42, no. 5, pp. 1050-1065, 2003.

[4] S. Wison, K. Foo, J. Cunningham et al., "Renal function and risk stratification in acute coronary syndromes," American Journal of Cardiology, vol. 91, no. 9, pp. 1051-1054, 2003.

[5] C. A. Herzog, R. W. Asinger, A. K. Berger et al., "Cardiovascular disease in chronic kidney disease. A clinical update from Kidney Disease: improving Global Outcomes (KDIGO)," Kidney International, vol. 80, no. 6, pp. 572-586, 2011.

[6] P. S. Tucker, A. T. Scanlan, and V. J. Dalbo, "Chronic kidney disease influences multiple systems: describing the relationship between oxidative stress, inflammation, kidney damage, and concomitant disease," Oxidative Medicine and Cellular Longevity, vol. 2015, Article ID 806358, 8 pages, 2015.

[7] M. Agharazii, R. St-Louis, A. Gautier-Bastien et al., "Inflammatory cytokines and reactive oxygen species as mediators of chronic kidney disease-related vascular calcification," American Journal of Hypertension, vol. 28, no. 6, pp. 746-755, 2015.

[8] M. Pruijm, B. Ponte, P. Vollenweider et al., "Not all inflammatory markers are linked to kidney function: results from a population-based study," American Journal of Nephrology, vol. 35, no. 3, pp. 288-294, 2012.

[9] Z. A. Massy, P. Stenvinkel, and T. B. Drueke, "The role of oxidative stress in chronic kidney disease," Seminars in Dialysis, vol. 22, no. 4, pp. 405-408, 2009.

[10] D. Fliser, "Asymmetric dimethylarginine (ADMA): the silent transition from an 'uraemic toxin' to a global cardiovascular risk molecule," European Journal of Clinical Investigation, vol. 35, no. 2, pp. 71-79, 2005.

[11] V. Liakopoulos, T. Eleftheriadis, T. Kyropoulos et al., "Hemodialysis procedure does not affect the levels of sICAM-1 and sVCAM-1 in patients with end stage renal disease," Renal Failure, vol. 27, no. 3, pp. 315-321, 2005.

[12] M. Sato, T. Konuma, N. Yanagisawa, H. Haizuka, H. Asakura, and Y. Nakashima, "Fas-Fas ligand system in the peripheral blood of patients with renal diseases," Nephron, vol. 85, no. 2, pp. 107-113, 2000.

[13] R. G. Fassett, S. K. Venuthurupalli, G. C. Gobe, J. S. Coombes, M. A. Cooper, and W. E. Hoy, "Biomarkers in chronic kidney disease: a review," Kidney International, vol. 80, no. 8, pp. 806821, 2011.

[14] J. Rysz, M. Banach, R. A. Stolarek et al., "Serum matrix metalloproteinases MMP-2 and MMP-9 and metalloproteinase tissue inhibitors TIMP-1 and TIMP-2 in diabetic nephropathy," Journal of Nephrology, vol. 20, no. 4, pp. 444-452, 2007.

[15] A. V. Fedulov, T. P. Ses, N. A. Gavrisheva et al., "Serum TGFbetal and TNF-alpha levels and cardiac fibrosis in experimental chronic renal failure," Immunological Investigations, vol. 34, no. 2, pp. 143-152, 2005.

[16] L. M. Blanco-Colio, J. L. Martín-Ventura, E. de Teresa et al., "Increased soluble Fas plasma levels in subjects at high cardiovascular risk: atorvastatin on inflammatory markers (AIM) study, a substudy of ACTFAST," Arteriosclerosis, Thrombosis, and Vascular Biology, vol. 27, no. 1, pp. 168-174, 2007.

[17] L. Del Vecchio and F. Locatelli, "Anemia in chronic kidney disease patients: treatment recommendations and emerging therapies," Expert Review of Hematology, vol. 7, no. 4, pp. 495506, 2014.

[18] P. K. Chatterjee, "Pleiotropic renal actions of erythropoietin," The Lancet, vol. 365, no. 9474, pp. 1890-1892, 2005.

[19] Y. Tanaka, N. Joki, H. Hase et al., "Effect of erythropoietinstimulating agent on uremic inflammation," Journal of Inflammation, vol. 9, no. 1, pp. 17-26, 2012.

[20] P. Bartnicki, P. Fijałkowski, M. Majczyk, J. Błaszczyk, M. Banach, and J. Rysz, "Effect of methoxy polyethylene glycolepoetin beta on oxidative stress in predialysis patients with chronic kidney disease," Medical Science Monitor, vol. 19, no. 8, pp. 954-959, 2013.

[21] P. Bartnicki, E. Majewska, M. Kowalczyk, Z. Baj, M. Banach, and J. Rysz, "Impact of anemia treatment with methoxy polyethylene glycol-epoetin beta on polymorphonuclear cells apoptosis in predialysis patients with chronic kidney disease," Pharmacological Reports, vol. 67, no. 5, pp. 842-845, 2015.

[22] P. Pozzoni, M. Pozzi, L. Del Vecchio, and F. Locatelli, "Epidemiology and prevention of cardiovascular complication in chronic kidney disease patients," Seminars in Nephrology, vol. 24, no. 5, pp. 417-422, 2004.

[23] B. J. G. Pereira, L. Shapiro, A. J. King, M. E. Falagas, J. A. Strom, and C. A. Dinarello, "Plasma levels of IL-1 $\beta$, TNF $\alpha$ and their specific inhibitors in undialyzed chronic renal failure, CAPD and hemodialysis patients," Kidney International, vol. 45, no. 3, pp. 890-896, 1994.

[24] C. Garlanda, C. A. Dinarello, and A. Mantovani, "The interleukin-1 family: back to the future," Immunity, vol. 39, no. 6, pp. 1003-1018, 2013.

[25] T. Higuchi, N. Fukuda, C. Yamamoto et al., "The influence of uremic serum on interleukin- $1 \beta$ and interleukin-1 receptor antagonist production by peripheral blood mononuclear cells," Therapeutic Apheresis and Dialysis, vol. 10, no. 1, pp. 65-71, 2006.

[26] J. Gupta, N. Mitra, P. A. Kanetsky et al., "Association between albuminuria, kidney function, and inflammatory biomarker profile in CKD in CRIC," Clinical Journal of the American Society of Nephrology, vol. 7, no. 12, pp. 1938-1946, 2012.

[27] B. T. Lee, F. A. Ahmed, L. L. Hamm et al., "Association of Creactive protein, tumor necrosis factor-alpha, and interleukin-6 with chronic kidney disease," BMC Nephrology, vol. 16, article 77, 2015.

[28] M. Bonomini, M. Reale, P. Santarelli, S. Stuard, N. Settefrati, and A. Albertazzi, "Serum levels of soluble adhesion molecules in chronic renal failure and dialysis patients," Nephron, vol. 79, no. 4, pp. 399-407, 1998.

[29] I. Kyriakos, V. S. Stel, E. Dounousi et al., "Inflammation, endothelial dysfunction and increased left ventricular mass in chronic kidney disease (CKD) patients: a longitudinal study," PLoS ONE, vol. 10, no. 9, Article ID e0138461, 2015.

[30] H. Tabibi, A. As'habi, M. Mahdavi-Mazdeh, M. Hedayati, and B. Nozary-Heshmati, "Comparison of novel risk factors for cardiovascular disease between hemodialysis patients with and without protein-energy wasting," International Urology and Nephrology, vol. 46, no. 10, pp. 2015-2020, 2014.

[31] J. Rysz, M. Banach, R. A. Stolarek et al., "Serum metalloproteinases MMP-2, MMP-9 and metalloproteinase tissue inhibitors TIMP-1 and TIMP-2 in patients on hemodialysis," International Urology and Nephrology, vol. 43, no. 2, pp. 491498, 2011.

[32] J. Rysz, E. Majewska, R. A. Stolarek, M. Banach, A. CiałkowskaRysz, and Z. Baj, "Increased levels of soluble TNF- $\alpha$ receptors 
and cellular adhesion molecules in patients undergoing bioincompatible hemodialysis," American Journal of Nephrology, vol. 26, no. 5, pp. 437-444, 2006.

[33] J. L. Martín-Ventura, L. M. Blanco-Colio, B. Muñoz-García et al., "NF- $\kappa \mathrm{B}$ activation and Fas ligand overexpression in blood and plaques of patients with carotid atherosclerosis: potential implication in plaque instability," Stroke, vol. 35, no. 2, pp. 458463, 2004.

[34] M. Nairz, T. Sonnweber, A. Schroll, I. Theurl, and G. Weiss, "The pleiotropic effects of erythropoietin in infection and inflammation," Microbes and Infection, vol. 14, no. 3, pp. 238246, 2012.

[35] D. S. Silverberg, D. Wexler, A. Iaina, and D. Schwartz, "The correction of anemia in patients with the combination of chronic kidney disease and congestive heart failure may prevent progression of both conditions," Clinical and Experimental Nephrology, vol. 13, no. 2, pp. 101-106, 2009.

[36] J.-Y. Choi, C. W. Yang, Y.-H. Kim et al., "Effect of conversion from ESA with shorter half-life to CERA once monthly for maintaining $\mathrm{Hb}$ concentration in pre-dialysis CKD patients," Kidney and Blood Pressure Research, vol. 37, no. 4-5, pp. 259$268,2013$.

[37] J. K. Inrig, S. K. Bryskin, U. D. Patel, M. Arcasoy, and L. A. Szczech, "Association between high-dose erythropoiesisstimulating agents, inflammatory biomarkers, and soluble erythropoietin receptors," BMC Nephrology, vol. 12, no. 1, pp. 67-77, 2011.

[38] G. Bellinghieri, C. G. Condemi, S. Saitta et al., "Erythropoiesisstimulating agents: dose and mortality risk," Journal of Renal Nutrition, vol. 25, no. 2, pp. 164-168, 2015.

[39] M. P. Curran and P. L. McCormack, "Methoxy polyethylene glycol-epoetin beta: a review of its use in the management of anaemia associated with chronic kidney disease," Drugs, vol. 68, no. 8, pp. 1139-1156, 2008.

[40] N. Shushakova, J. K. Park, J. Menne, and D. Fliser, "Chronic erythropoietin treatment affects different molecular pathways of diabetic cardiomyopathy in mouse," European Journal of Clinical Investigation, vol. 39, no. 9, pp. 755-760, 2009. 


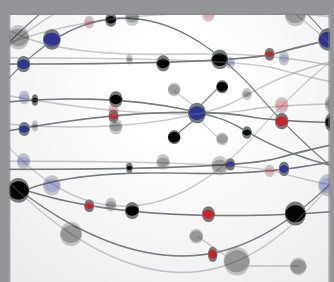

The Scientific World Journal
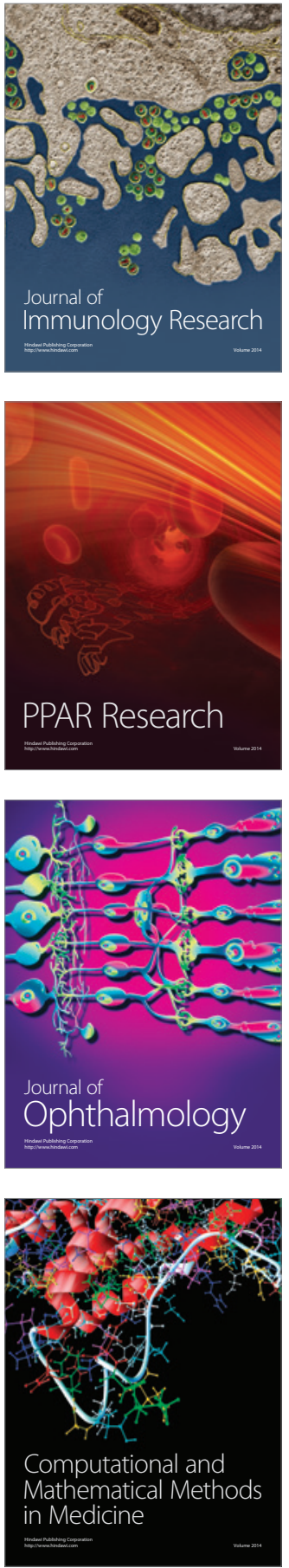

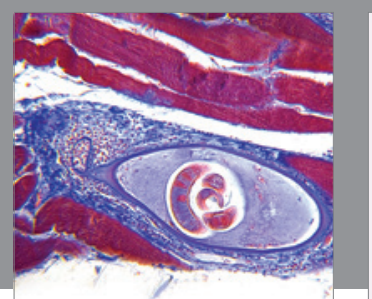

Gastroenterology Research and Practice

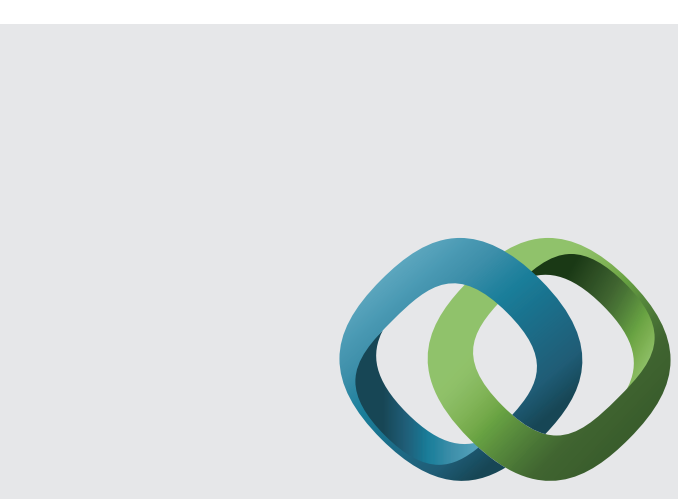

\section{Hindawi}

Submit your manuscripts at

http://www.hindawi.com
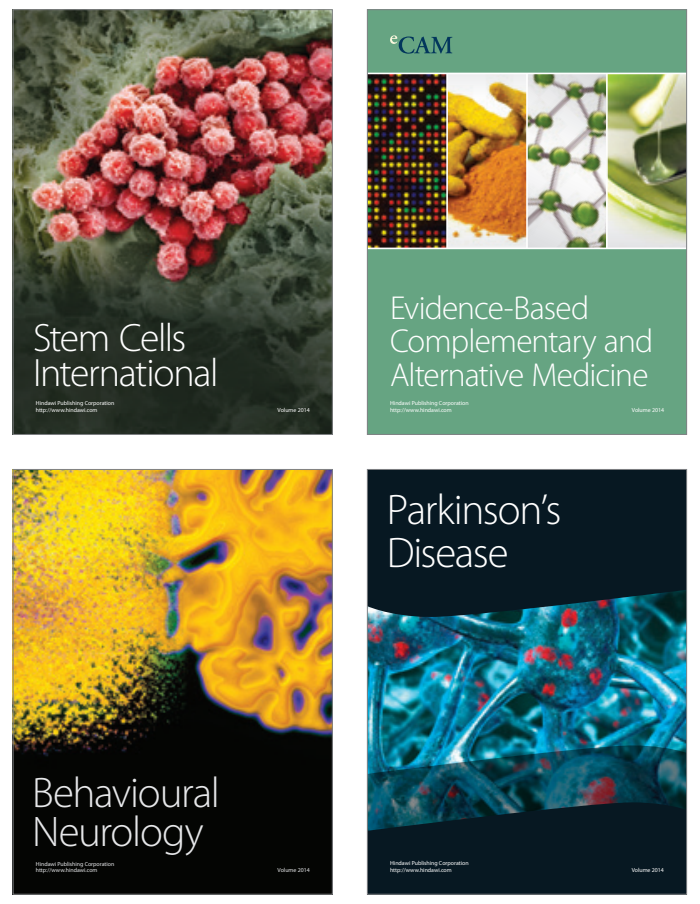
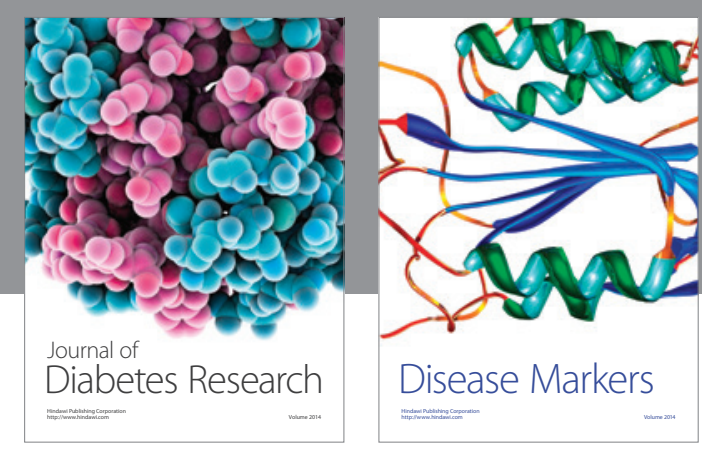

Disease Markers
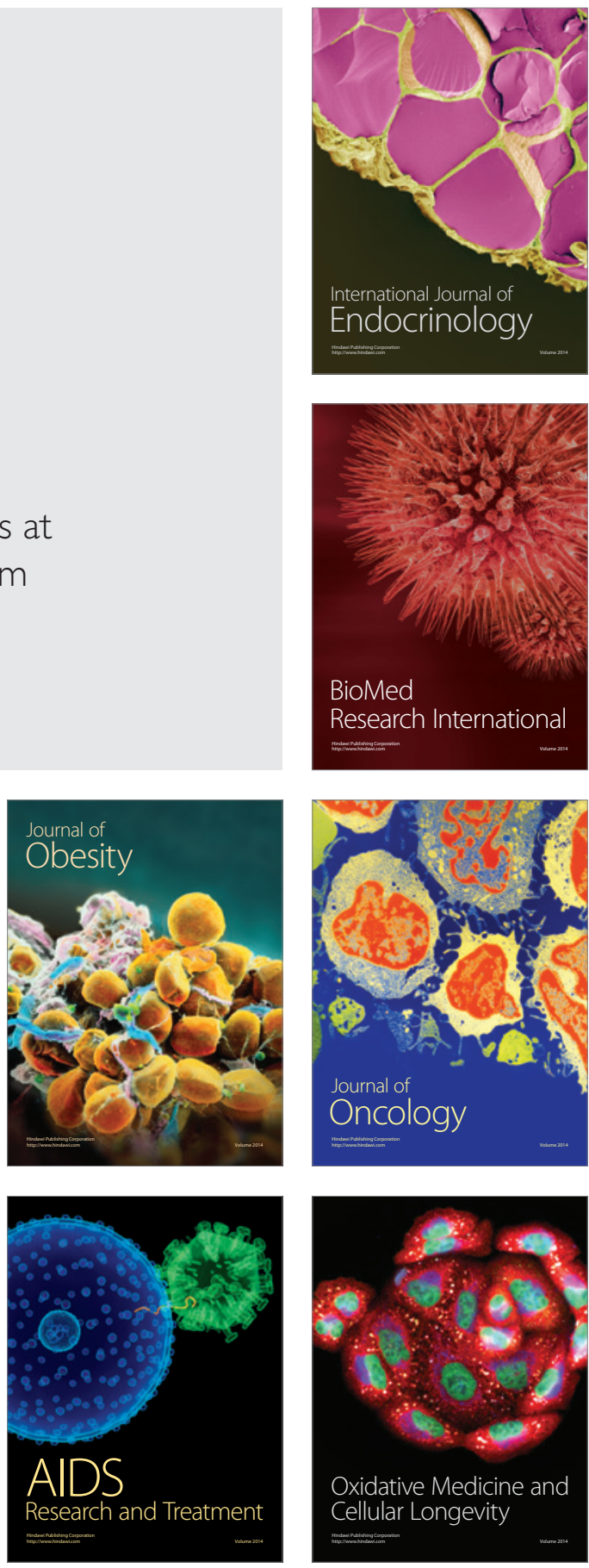